\title{
Calcium Release in Response to Local Nanomechancial Shear Stress Delivered by an AFM Tip
}

\author{
Daniel J. Modulevsky ${ }^{1}$, Nickolay V. Bukoreshtliev ${ }^{1}$ and Andrew E. Pelling ${ }^{1,2,3}$ \\ 1. Department of Physics, University of Ottawa, Ottawa, Canada \\ 2. Department of Biology, University of Ottawa, Ottawa, Canada \\ 3. Institute for Science, Society and Policy, University of Ottawa, Ottawa, Canada
}

The Atomic Force Microscope (AFM) [1] has found considerable utility in the study of biological molecules and cells. The combination of AFM with other imaging modalities, such as laser scanning confocal microscopy (LSCM) [2], has allowed researchers to investigate the biochemical response of cells to local nanoscale forces by employing fluorescent fusion proteins of live cell fluorophores. Fluo-4 AM is a commonly employed calcium indicator that becomes fluorescent when bound to calcium cations. Previous studies have shown that osteoblast cells can be stimulated by AFM tip indentation when touching down or lifting off the cell in a force-dependent fashion. The strain caused by the AFM tip opens stretch activated channels that ultimately lead to cell depolarization. In turn, this causes the release of intracellular calcium stores and an observable change in fluorescence intensity. This phenomenon is not limited to osteoblasts as mechanical deformation can lead to the release of intracellular calcium stores in several cell types [3-5].

In addition, cells cultured on flexible substrates have also been observed to release intra-cellular calcium when exposed to local substrate stretching. Fibroblasts exposed to a small equiaxial stretch were observed to initially release calcium and then exhibit subsequent calcium oscillations after membrane relaxation [6]. In a recent study, a micro-indenter was used to locally deform and vibrate a region of a flexible membrane within $13 \mu \mathrm{m}$ of the cell edge at a frequency of $140 \mathrm{~Hz}$. In this case, both local and global intra-cellular calcium release was observed in response to localized membrane stretch near to the cell edge. [7] These studies suggest that a multitude of mechanical stimuli that can result in the release of intracellular calcium stores. This phenomenon is important as calcium signaling is hypothesized to be a major intra- and inter-cellular signaling mechanism [8]. Significantly, this implies that a common calcium signaling cascade can be employed in response to very different mechanical stimuli.

Here, we performed simultaneous AFM-LSCM experiments on monolayers of HeLa epithelial cells loaded with the Fluo-4 AM calcium indicator. Interestingly, unlike previous studies, no intracellular calcium release was observed to take place when the AFM tip touched down or lifted off the cells with applied forces ranging from 1-15 $\mathrm{nN}$. However, we also performed an additional experiment in which the AFM tip was used to apply a shear stress to cell membranes, as opposed to applying a normal force. In this experiment, the AFM tip was moved laterally across a monolayer of cells (typically a distance of $\sim 100 \mu \mathrm{m})$ with an applied force of 1-20 $\mathrm{nN}$ and speeds ranging from 1-10 $\mu \mathrm{m} / \mathrm{sec}$. Application of a shear stress was observed to clearly result in the release of intracellular calcium stores and a resulting increase in fluorescence intensity. Furthermore, the calcium signal propagated to surrounding cells from the initial stimulated cell. Cells could be stimulated repeatedly and calcium release displayed a clear sensitivity to the state of the cytoskeleton and shear stress characteristics. Our findings suggest that Hela cells possess a mechanism of calcium release that is sensitive to membrane stretch that results from lateral strain as opposed to normal strain. 

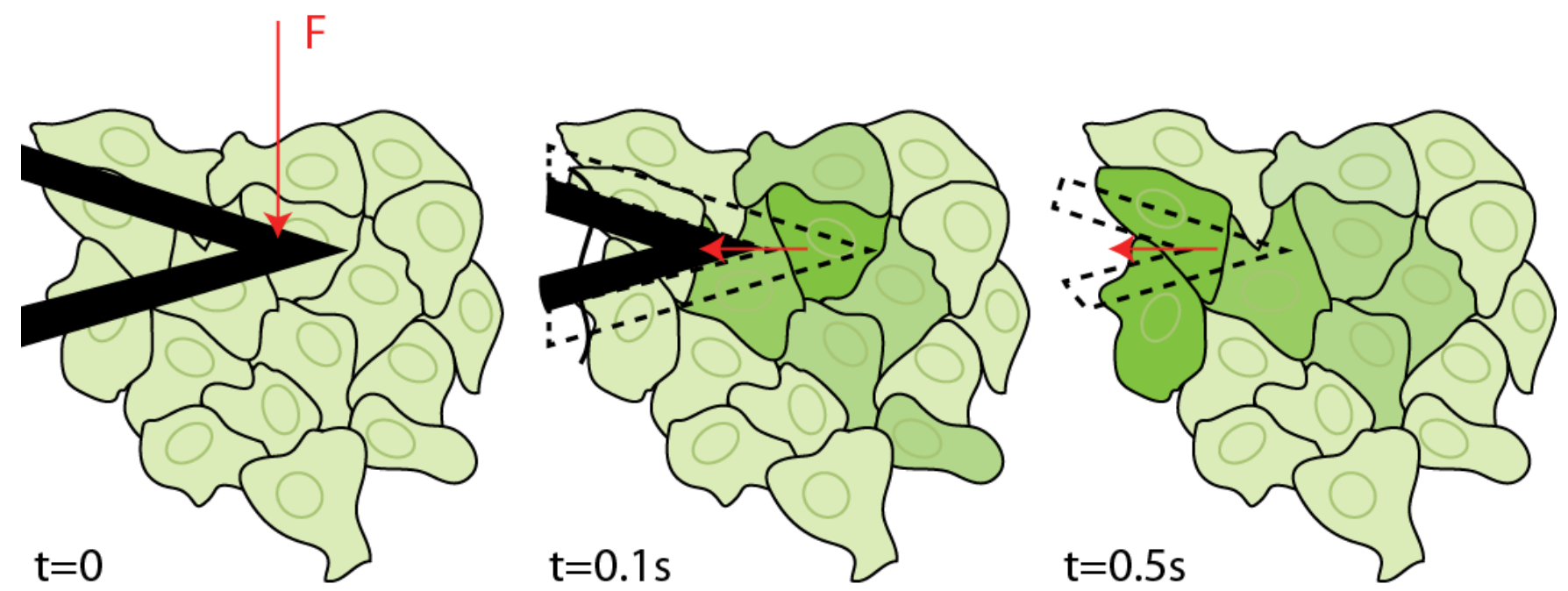

Figure 1. Experimental Approach: A cluster of HeLa cells stained with Fluo-4 AM. The AFM cantilever is brought into contact with the cells at a defined force and moved to the left (red arrows) at a constant velocity. The resulting shear stress stimulates an intracellular release of calcium causing a localized increase in fluorescence intensity.

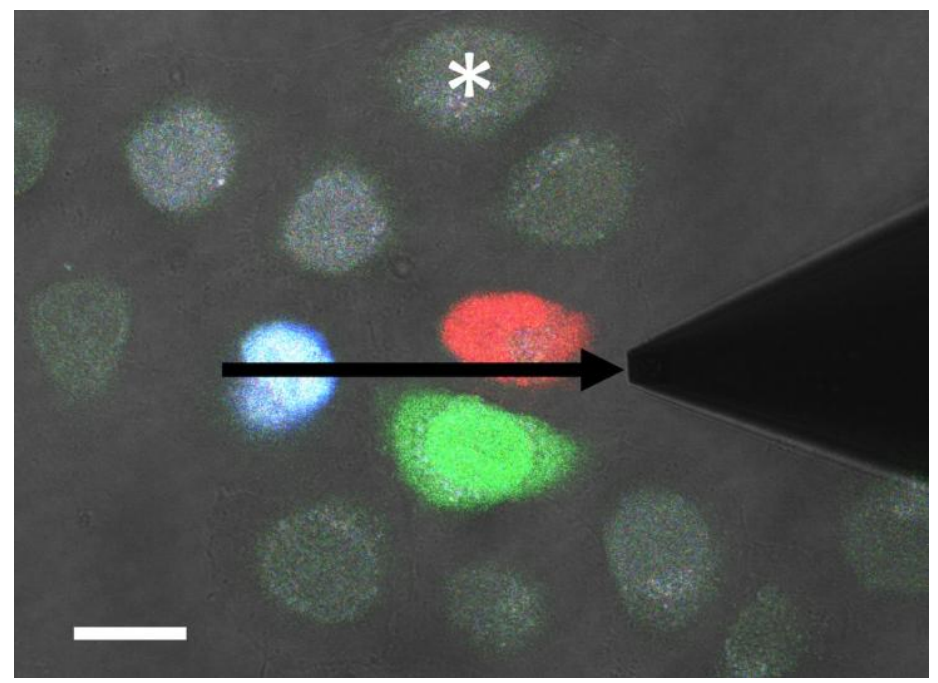

Figure 2. Calcium release in response to shear stress. The AFM tip is moved a distance of $100 \mu \mathrm{m}$ across a group of cells at $1 \mu \mathrm{m} / \mathrm{sec}$ along a linear path (black arrow). This applies a shear stress to different parts of each cell but always results in a sudden release of intracellular calcium compared to basal levels (asterisk). Increases in fluorescence intensity are colored according to the time of stimulation in each cell - 0sec (blue), 20sec (green) and $40 \mathrm{sec}$ (red). Scale bar $=20 \mu \mathrm{m}$.

\section{References:}

[1] J Heinisch, et al, Journal of Cell Science 125 (2012), p. 4189-95.

[2] L Guolla, et al, Journal of Cell Science 125 (2012), p. 603-13.

[3] BF Jones, et al, Journal of Biomechanics 38 (2005), p. 1653-64.

[4] TA Lusardi, et al, Journal of Neurotrauma 21 (2004), p. 61-72

[5] KK Ceelen, et al, Experimental Mechanics 49 (2007), p. 25-33

[6] PD Arora, KJ Bibby, CA McCulloch, Journal of Cellular Physiology 161 (1994), p. 187-200

[7] WS Nishitani, TA Saif, Y Wang, Plos One 6 (2011), p. e26181

[8] NV Bukoreshtliev, K. Haase, AE Pelling, Cell and Tissue Research (2012), In press.

[9] Acknowledgements: NSERC Discovery and Discovery Accelerator Grants, Province of Ontario Early Researcher Award, Canada Research Chairs Program 